\title{
Unilateral versus bilateral endoscopic stenting in patients with unresectable malignant hilar obstruction: a systematic review and meta-analysis
}

다 (i)

\author{
Authors \\ Institutions \\ 1 Division of Gastroenterology and Hepatology, Johns \\ Hopkins Medical Institutions, Baltimore, Maryland, \\ United States \\ 2 Russell H. Morgan Department of Radiology and \\ Radiological Sciences, Johns Hopkins University, \\ Baltimore, Maryland, United States \\ 3 Welch Medical Library, Johns Hopkins University, \\ Baltimore, Maryland, United States
}

Mohamad Aghaie Meybodi ${ }^{1}$, Delaram Shakoor ${ }^{2}$, Julie Nanavati ${ }^{3}$, Yervant Ichkhanian ${ }^{1}$, Kia Vosoughi ${ }^{1}$, Olaya I. Brewer Gutierrez ${ }^{1}$, Anthony N. Kalloo', Vikesh Singh ${ }^{1}$, Vivek Kumbhari ${ }^{1}$, Saowanee Ngamruengphong ${ }^{1}$, Mouen A. Khashab ${ }^{1}$

submitted 31.7.2019

accepted after revision $\quad 16.10 .2019$

\author{
Bibliography \\ DOI https://doi.org/10.1055/a-1067-4326 | \\ Endoscopy International Open 2020; 08: E281-E290 \\ (c) Georg Thieme Verlag KG Stuttgart · New York \\ eISSN 2196-9736
}

\section{Corresponding author}

Mouen Khashab MD, Associate Professor of Medicine, Director of Therapeutic Endoscopy, Division of

Gastroenterology and Hepatology, Johns Hopkins Medical Institutions, 1800 Orleans St, Sheikh Zayad 7E Rm 7125G, Baltimore, MD 21224

Fax: +1-443-287-1960

mkhasha1@jhmi.edu $\circledast$ Supplementary material

Online content viewable at:

https://doi.org/10.1055/a-1067-4326

\section{ABSTRACT}

Background and study aims Endoscopic stent placement is used for palliative management of unresectable malignant hilar obstruction, which could be achieved by either unilateral or bilateral stent insertion.

Materials and methods A literature search was performed to identify studies that reported outcomes of metallic biliary stent placement in patients with malignant hilar obstruction. Weighted pooled rates (WPR) along with $95 \%$ confidence intervals $(95 \% \mathrm{Cl})$ were calculated to determine and compare outcomes including technical and functional success, early and late adverse events, post procedure cholangitis, and stent occlusion between two groups. Results A total of 21 studies with 1292 patients were included. WPR of technical success was significantly higher in the unilateral group ( $97 \%, 95 \% \mathrm{Cl}: 93-98 \%)$ vs. bilateral group $(89 \%, 95 \% \mathrm{Cl}: 84-92 \%)(P=0.0 .003)$. WPR for functional success in the unilateral and bilateral groups were $96 \%$ (95\%Cl: $91-98 \%)$ and $94 \%(95 \% \mathrm{Cl}: 91-97 \%)$, respectively $(P=0.48)$. The rate of early and late complications was comparable between the two groups.

Conclusion In patients with unresectable malignant hilar obstruction, unilateral and bilateral metallic stenting techniques are comparable in terms of efficacy and safety.

\section{Introduction}

Unresectable malignant hilar biliary obstruction could be caused by a wide variety of neoplasms [1,2]. Certain malignancies such as Klatskin tumor or hilar cholangiocarcinoma can lead to intrinsic obstruction, while other tumors, including gallbladder carcinoma, hepatomas, pancreatic adenocarcinoma, and metastases contribute to extrinsic obstruction, which ultimately lead to obstructive jaundice $[3,4]$.

Thus, palliative biliary drainage is absolutely crucial for improving the quality of life of patients with unresectable malig- nant hilar biliary obstruction. Percutaneous or endoscopic drainage as well as surgical bypass are currently the mainstay of palliative treatment $[5,6]$. Due to the limited survival of these patients, minimally-invasive procedures such as endoscopic drainage may be more appealing. Furthermore, prior studies have shown that comparing to surgical bypass procedures, endoscopic drainage could provide better outcomes and lower complication rate in the palliative management of patients with unresectable malignant hilar obstruction [7,8].

Endoscopic drainage in patients with unresectable malignant hilar obstruction entails using a plastic or self-expandable 
metal stent (SEMS). Despite similar technical success rates, metallic stents have been shown to provide longer patency than their plastic counterpart [6]; however, there is no consensus regarding the best treatment option for palliative treatment of patients with unresectable hilar obstruction. In addition, debate remains on the superiority of bilateral stenting over unilateral stenting. While in unilateral stenting there are concerns over suboptimal efficacy in relieving jaundice and bacterial contamination of the undrained segment $[9,10]$, the technical complexity of bilateral stenting has been a major drawback [11]. Therefore, given conflicting outcomes with different endoscopic strategies, we performed a systematic review and meta-analysis to determine the overall technical and functional success rates, as well as the adverse event $(\mathrm{AE})$ rate of unilateral and bilateral metallic stenting and to compare outcomes in management of patients with unresectable malignant hilar obstruction.

\section{Materials and methods}

This systematic review was performed in accordance with the guidelines of the Preferred Reporting Items for Systematic Reviews and Meta-Analyses (PRISMA) [12] and meta-analysis of observational studies in epidemiology (MOOSE) [13].

\section{Literature search and study selection}

A comprehensive literature search strategy was developed by an experienced medical reference librarian (JN). Four electronic databases - Medline, Embase, Cochrane, and ISI Web of Sciences - were screened to identify relevant studies published through June 1, 2018. The following MeSH and keyword search terms were used: "unilateral stent or drainage, bilateral stent or drainage, malignant hilar obstruction, Klatskin tumor, biliary tract neoplasm, gallbladder tumor". Literature search was conducted by two investigators (MAM, DS), independently. The retrieved records were screened on the basis of the title and abstract, and the eligible articles were obtained for full-text review. Only English language articles were included. The bibliography of retrieved articles were manually reviewed to reveal any additional relevant studies.

\section{Inclusion and exclusion criteria}

Inclusion criteria were determined by two investigators (MAM, MAK). Studies were eligible for inclusion if they described outcomes of unilateral or bilateral endoscopic hepatic duct drainage by SEMS in management of patients with unresectable malignant hilar obstruction. Randomized clinical trials (RCTs) or observational studies that reported technical and functional success rates as well as early and late AEs. Technical success was defined as successful stent insertion across the hilar stricture. Functional success was defined as $50 \%$ decrease in bilirubin level within 14 days or a $75 \%$ decrease in bilirubin level within a month. Early AEs were defined as any procedure-related complications that occurred within 30 days, while late AEs were described as complications occurring later than 30 days. Cholangitis was reported if the patient had fever, leukocytosis, and increasing bilirubin. Stent occlusion was defined as increase in bilirubin, recurrence of jaundice, and presence of imaging findings suggestive of intrahepatic bile duct dilation.

Individual case reports or case series with 10 or fewer patients were excluded. Published abstracts of national or international conferences were not included in the study because assessment of risk of bias in them was not possible. Studies that only reported outcomes of plastic stenting were also excluded. Titles and abstracts of obtained records were screened by two investigators (MAM, DS), independently. All eligible articles were downloaded into EndNote 7.0 (Thomson ISI ResearchSoft, Philadelphia, Pennsylvania, United States) and duplicate studies were removed. For final inclusion, eligible studies were reviewed at the full-text level to determine their eligibility. Any disagreement was resolved through discussion with the senior author (MAK).

\section{Data extraction and quality assessment}

The following data were extracted from the included studies: study design, year of publication, number of patients, age, sex, type of stent (plastic or SEMS), stenting strategy (unilateral or bilateral), technical success rate, functional success rate, occlusion rate, early and late AEs, post-procedure cholangitis, stent patency, survival and follow-up duration. Two investigators (MAM, DS), independently assessed the quality of included studies using the National Institutes of Health (NIH) quality assessment tool for before-after (pre-post) studies with no control group and the Cochrane tool for RCTs [14].

\section{Statistical analysis and data synthesis}

Weighted pooled rates (WPR) along with their $95 \%$ confidence intervals $(\mathrm{Cl})$ and predictive interval were calculated for technical success, functional success, early and late AEs, post-procedure cholangitis, and stent occlusion. The weight of each study was calculated by the inverse of its variance. Subgroup analysis was performed to compare effect size in studies that employed unilateral or bilateral stent. Cochran $\mathrm{Q}$ test and $\mathrm{I}^{2}$ were assessed for heterogeneity across studies [15]. Presence of significant heterogeneity was considered when $P<0.1$ for the Cochrane $Q$ test. The $\mathrm{I}^{2}$ values of $0-50 \%, 50 \%$ to $75 \%$, and $75 \%$ to $100 \%$ were interpreted as low, moderate, and high level of heterogeneity, respectively [16]. Due to heterogeneity between types and sizes of stents, DerSimonian-Laird random-effects model of meta-analysis was applied $[17,18]$. A random effects model was also used to combine studies within each subgroup. $P<$ 0.05 was considered significant.

A sensitivity analysis was performed to determine if one-arm studies had a disparate effect on the pooled effect. We calculated the pooled effect with only two-arm studies. With this approach, if the pooled effect does not change significantly, then the one-arm studies do not disturb the pooled effect. Visual evaluation of funnel plots and the Begg-Mazumdar regression test were performed for evaluation of presence of publication bias [19]. If publication bias was present, Duval and Tweedie's "trim and fill" method was used to adjust effect size, accordingly. All analysis was performed by one investigator (MAM), using Comprehensive Meta-Analysis software (version 2.0; Biostat; Englewood, New Jersey, United States). 


\section{Results}

\section{Literature search}

Based on our search strategy, we identified 35 records from PubMed, 49 records from Embase, 22 records from Cochrane, and 47 records from ISI Web of Science. Fifty-six duplicate records were removed and an additional 63 studies were excluded after screening titles and abstracts. Thirty-four studies were selected for the full-text review and 16 studies were excluded due to our exclusion criteria; one study [20] was excluded due to serial insertion of stents and another study [21] was excluded due to possible population overlap with another included study [22]. Finally, 18 studies with 911 patients were included in our meta-analysis. Our search strategy and study selection are summarized in a PRISMA flowchart ( $\mathbf{F i g . 1}$ ).

\section{Study characteristics and quality assessment}

Among included studies, 10 [22-31] were retrospective and the rest [32-39] were prospective. Two studies [32,33] were RCTs. Thirteen studies $[23-31,34,35,38,39]$ were single-center and the other five studies [21, 32, 33, 36, 37] were multicenter. Two studies $[38,39]$ evaluated only unilateral stents, 11 studies $[21,23,25-27,30,31,34-37]$ evaluated only bilateral stents and five studies $[24,28,29,32,33]$ compared unilateral vs. bilateral stents.

Among 911 patients with malignant hilar obstruction, cholangiocarcinoma was the most frequent etiology (70\%). Seventeen percent of patients had stricture due to gallbladder carcinoma, and $13 \%$ of patients had obstruction related to other causes. One study [39] did not report the etiology. According to the Bismuth classification, $29.25 \%$ of cases were type II, $38.5 \%$ of cases were type III, and $32.25 \%$ of cases were type IV. Two studies $[23,28]$ did not report Bismuth classification. Demographic data and study characteristics of included studies are described in $\triangleright$ Table $\mathbf{1}$ and $\boldsymbol{\vee T a b l e ~} \mathbf{2}$.

The Cochrane tool for assessing risk of bias was used for quality assessment of the two RCTs. Performance bias was high due to awareness of endoscopists and patients about stent type; however, risk of bias in other domains, including selection, detection, attrition, and reporting was low. Quality assessment of the observational studies was done by $\mathrm{NIH}$ quality assessment tool for Before-After (Pre-Post) Studies With No Control Group. Nine studies $[24,25,28-31,34,35,38]$ had good quality and seven studies $[23,26,27,32,36,37,39]$ had fair quality ( $\triangleright$ Table 3$)$.

\section{Meta-analysis}

Unilateral vs. bilateral metallic stent

Among included studies, 18 [22-39] recruited the self-expandable metallic stent. The weighted pooled rate (WPR) of technical success was $91 \%(95 \% \mathrm{Cl}: 88-94 \%$, prediction interval: $72-98 \%)$, Cochrane $Q$ test $P=0.001, I^{2}=55 \%$. The Begg-Mazumdar test revealed no publication bias (tau $=0.20, P=0.17$, two-tailed) (Supplementary Fig.1). Subgroup analysis was used to compare effect size between unilateral and bilateral
PubMed: 35;

Embase: 49;

Cochrane: 22;

Web of Science: 47; $(N=153)$

56 records removed as duplicates

Title and abstracts of all identified records were screened $(\mathrm{N}=97)$

63 records excluded after title and abstract review

Eligible studies were assessed at the level of their full text $(n=34)$

Full-text articles excluded $(n=16)$

- Used only plastic stent: 3

- Review article: 5

- Studies with no relevant data: 4

- Study with less than 10 cases: 3

- Studies with overlap population: 1

18 studies included for final meta-analysis

- Fig. 1 PRISMA flowchart. From: Moher D, Liberati A, Tetzlaff J, Altman DG, The PRISMA Group (2009). Preferred Reporting Items for Systematic Reviews and Meta-Analyses: The PRISMA Statement. PLoS Med 6(7): e1000097. doi:10.1371/journal.pmed1000097.

stenting. The technical success rate was $97 \%(95 \% \mathrm{Cl}$ : $93-98 \%$, prediction interval: $91-97 \%)$ and $89 \%(95 \% \mathrm{Cl}: 84-92 \%$, prediction variable: $69-97 \%$ ) in the unilateral and bilateral stenting, respectively. The technical success rate was significantly higher in patients who underwent unilateral metallic stenting as compared to those who underwent bilateral metallic stenting $(P=0.003)$ ( $\mathbf{F i g . 2 a )}$. Sensitivity analysis by pooling data from two-arms studies also showed the higher technical success rate in unilateral group $(R R=3.82, P=0.03)$.

The WPR of functional success was $95 \%(95 \% \mathrm{Cl}$ : $92-96 \%$, predictive interval: $87-98 \%$ ), Cochrane $Q$ test $P=0.24, \mathrm{I}^{2}=$ $18 \%$. There was no publication bias according to the Begg-Mazumdar regression test (tau $=0.05, P=0.74$, two-tailed). The WPR of functional success of unilateral stenting was $96 \%$ (95\% Cl: $90-98 \%$, predictive interval: 84-99\%) and bilateral stenting was $94 \%$ (95\% Cl: $91-96 \%$, predictive interval: 79-98\%). There was no statistically significant difference between the functional success rate of unilateral and bilateral stenting $(P=0.481)$ ( $\vee$ Fig. 2b). Sensitivity analysis also showed no statistically significant difference between two groups ( $R R=2.18, P=0.22)$.

In total, 60 early complications happened in the 517 patients. The WPRs of early complication were $15 \%(95 \% \mathrm{Cl}: 7-$ $30 \%)$ and $9 \%(95 \% \mathrm{Cl}: 6-15 \%)$ in unilateral and bilateral SEMS, respectively ( $\triangleright$ Fig.3a). Cholangitis was the most common type 
- Table 1 Demographic and technical data from single-arm studies.

\begin{tabular}{|c|c|c|c|c|c|c|c|c|}
\hline Study & Stent & $\begin{array}{l}\text { Number of } \\
\text { patients } \\
\text { (male) }\end{array}$ & $\begin{array}{l}\text { Age } \\
(\text { mean } \pm \text { SD) }\end{array}$ & $\begin{array}{l}\text { Technical } \\
\text { success, \% }\end{array}$ & $\begin{array}{l}\text { Functional } \\
\text { success, \% }\end{array}$ & $\begin{array}{l}\text { Stent paten- } \\
\text { cy, (day, } \\
\text { median) }\end{array}$ & $\begin{array}{l}\text { Survival, } \\
\text { (day, } \\
\text { median) }\end{array}$ & Follow-up \\
\hline $\begin{array}{l}\text { De Palma } \\
\text { et al } 2003\end{array}$ & Unilateral stent & $61(40)$ & $62.5 \pm 11.2$ & 96.7 & 100 & 169 & 140 & $\mathrm{~N} / \mathrm{A}$ \\
\hline $\begin{array}{l}\text { Singh et al } \\
2004\end{array}$ & Unilateral stent & $18(7)$ & $53.7 \pm 11.1$ & 100 & 100 & $\mathrm{~N} / \mathrm{A}$ & $\mathrm{N} / \mathrm{A}$ & $\mathrm{N} / \mathrm{A}$ \\
\hline $\begin{array}{l}\text { Chahal } \\
\text { et al } 2010\end{array}$ & Bilateral stent & $21(15)$ & $63.7 \pm 13.9$ & 100 & $\mathrm{~N} / \mathrm{A}$ & 189 & N/A & $\begin{array}{l}6.14 \\
\text { months }\end{array}$ \\
\hline $\begin{array}{l}\text { Hwang } \\
\text { et al } 2011\end{array}$ & Bilateral stent & $30(20)$ & $68 \pm 8$ & 86.7 & 100 & 140 & 176 & 5.8 months \\
\hline $\begin{array}{l}\text { Kim et al } \\
2012\end{array}$ & Bilateral stent & $97(48)$ & 71 median & 78.4 & 97.4 & 159 & 226 & 234 days \\
\hline $\begin{array}{l}\text { Kim et al } \\
2009\end{array}$ & Bilateral stent & $34(19)$ & 68.3 median & 85.3 & 100 & 186 & 239 & 21 months \\
\hline $\begin{array}{l}\text { Kitamura } \\
\text { et al } 2017\end{array}$ & Bilateral stent & $17(9)$ & 78 median & 100 & 82 & $\mathrm{~N} / \mathrm{A}$ & $\mathrm{N} / \mathrm{A}$ & 192 days \\
\hline $\begin{array}{l}\text { Law et al } \\
2013\end{array}$ & Bilateral stent & $24(19)$ & $68 \pm 13$ & 100 & $\mathrm{~N} / \mathrm{A}$ & $\mathrm{N} / \mathrm{A}$ & $\mathrm{N} / \mathrm{A}$ & 97 days \\
\hline $\begin{array}{l}\text { Lee et al } \\
2013\end{array}$ & Bilateral stent & $84(48)$ & $68.3 \pm 15.8$ & 95.2 & 97.5 & 238 & 256 & $\mathrm{~N} / \mathrm{A}$ \\
\hline $\begin{array}{l}\text { Park et al } \\
2016\end{array}$ & Bilateral stent & $31(16)$ & $67 \pm 14$ & 83.9 & 92.3 & 188 & 175 & $\mathrm{~N} / \mathrm{A}$ \\
\hline $\begin{array}{l}\text { Park et al } \\
2009\end{array}$ & Bilateral stent & $35(21)$ & 66 & 94.3 & 100 & 150 & 180 & 142 days \\
\hline $\begin{array}{l}\text { Yang et al } \\
2018\end{array}$ & Bilateral stent & $43(22)$ & $72.9 \pm 9.1$ & 88.4 & 100 & 198 & 300 & 184 days \\
\hline $\begin{array}{l}\text { Dumas } \\
\text { et al } 2000\end{array}$ & Bilateral stent & $45(28)$ & 72 & 73.3 & 100 & $\mathrm{~N} / \mathrm{A}$ & $\mathrm{N} / \mathrm{A}$ & 8.5 months \\
\hline
\end{tabular}

- Table 2 Demographic and technical data from two-arm studies.

\begin{tabular}{|c|c|c|c|c|c|c|c|c|c|c|c|c|}
\hline \multirow[t]{2}{*}{ Study } & \multicolumn{2}{|c|}{$\begin{array}{l}\text { Number of } \\
\text { patients (male) }\end{array}$} & \multicolumn{2}{|l|}{ age } & \multicolumn{2}{|c|}{$\begin{array}{l}\text { Technical } \\
\text { success\% }\end{array}$} & \multicolumn{2}{|c|}{$\begin{array}{l}\text { Functional } \\
\text { success\% }\end{array}$} & \multicolumn{2}{|c|}{$\begin{array}{l}\text { Stent patency, } \\
\text { (day, median) }\end{array}$} & \multicolumn{2}{|c|}{$\begin{array}{l}\text { Survival, } \\
\text { (day, median) }\end{array}$} \\
\hline & Uni & $\mathbf{B i}$ & Uni & $\mathbf{B i}$ & Uni & $\mathbf{B i}$ & Uni & $\mathbf{B i}$ & Uni & $\mathrm{Bi}$ & Uni & $\mathbf{B i}$ \\
\hline $\begin{array}{l}\text { Lee et al } \\
2017\end{array}$ & $66(33)$ & $67(36)$ & $74.1 \pm 10.42$ & $73.5 \pm 10.42$ & 100 & 95.5 & 95.3 & 87.5 & 139 & 252 & 178 & 270 \\
\hline $\begin{array}{l}\text { Iwano et al } \\
2011\end{array}$ & $63(35)$ & $19(11)$ & 71.6 & 66.6 & 95.2 & 89.4 & $\mathrm{~N} / \mathrm{A}$ & $\mathrm{N} / \mathrm{A}$ & 133 & 125 & 170 & 184 \\
\hline $\begin{array}{l}\text { Naitoh et al } \\
2009\end{array}$ & $17(9)$ & $29(12)$ & $69 \pm 14$ & $70 \pm 11$ & 100 & 90 & 94.1 & 96.1 & 210 & 488 & 166 & 205 \\
\hline $\begin{array}{l}\text { Mukai et al } \\
2012\end{array}$ & 14 & 16 & $\mathrm{~N} / \mathrm{A}$ & $\mathrm{N} / \mathrm{A}$ & 100 & 100 & 100 & 100 & 363 & 295 & $\mathrm{~N} / \mathrm{A}$ & $\mathrm{N} / \mathrm{A}$ \\
\hline $\begin{array}{l}\text { Liberato } \\
\text { et al } 2012\end{array}$ & 35 & 45 & $\mathrm{~N} / \mathrm{A}$ & $\mathrm{N} / \mathrm{A}$ & 100 & 93.3 & $\mathrm{~N} / \mathrm{A}$ & $\mathrm{N} / \mathrm{A}$ & $\begin{array}{l}24 \\
\text { weeks }\end{array}$ & $\begin{array}{l}29 \\
\text { weeks }\end{array}$ & $\mathrm{N} / \mathrm{A}$ & $\mathrm{N} / \mathrm{A}$ \\
\hline
\end{tabular}




\begin{tabular}{|c|c|c|c|c|c|c|c|c|}
\hline 总 $\bar{\square}$ & 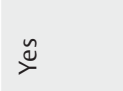 & 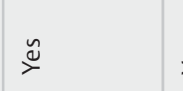 & $\stackrel{\check{\nu}}{\check{\nu}}$ & $\stackrel{\mathscr{\nu}}{\circlearrowright}$ & $\stackrel{\circlearrowright}{\circlearrowright}$ & 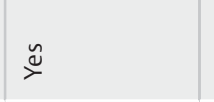 & $\stackrel{\Perp}{\varpi}$ & $\stackrel{\circ}{z}$ \\
\hline 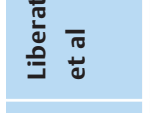 & 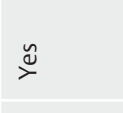 & 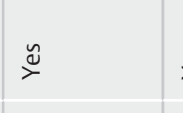 & ֻِ & $\stackrel{\infty}{0}$ & 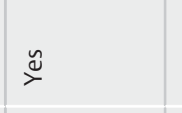 & 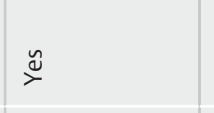 & $\stackrel{y}{2}$ & $\stackrel{8}{2}$ \\
\hline 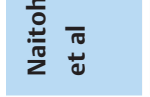 & 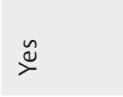 & $\stackrel{\check{\nu}}{\check{\nu}}$ & ֻِّ & 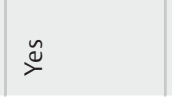 & 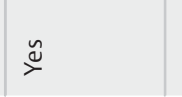 & 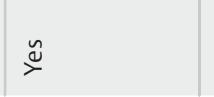 & 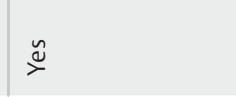 & z \\
\hline 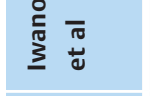 & $\stackrel{0}{0}$ & $\stackrel{\infty}{\nu}$ & 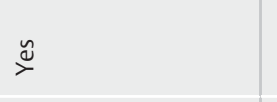 & 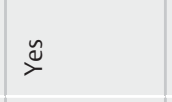 & 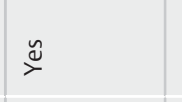 & 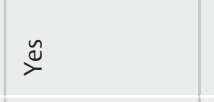 & 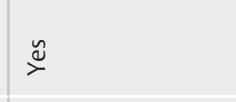 & $\stackrel{̊}{z}$ \\
\hline 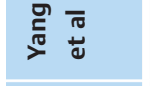 & $\stackrel{\circ}{\check{\nu}}$ & $\stackrel{y}{x}$ & $\stackrel{\check{\nu}}{\check{\nu}}$ & $\stackrel{y}{\check{\nu}}$ & $\stackrel{\check{\nu}}{\check{\nu}}$ & $\stackrel{\check{\nu}}{\check{\nu}}$ & $\stackrel{\mathscr{D}}{x}$ & $\stackrel{\imath}{z}$ \\
\hline 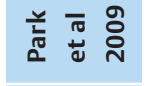 & $\stackrel{0}{\check{\nu}}$ & $\stackrel{\Xi}{\check{D}}$ & 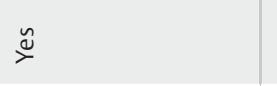 & 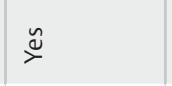 & 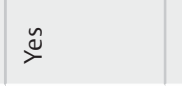 & 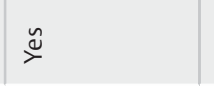 & 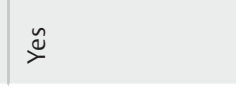 & $\stackrel{̊}{2}$ \\
\hline 言 অ & $\check{\check{\nu}}$ & 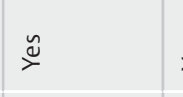 & 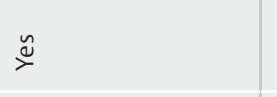 & 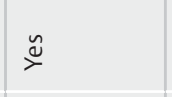 & 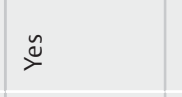 & 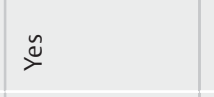 & 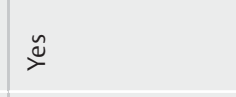 & $\stackrel{̊}{2}$ \\
\hline 巳 ฐ & 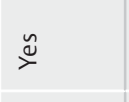 & 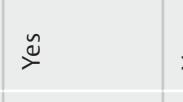 & $\stackrel{\check{\nu}}{\check{\nu}}$ & 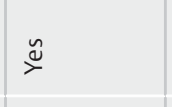 & 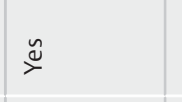 & 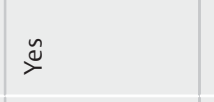 & $\stackrel{\Xi}{\check{\nu}}$ & $\stackrel{2}{z}$ \\
\hline 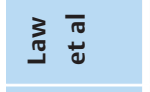 & 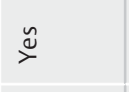 & $\stackrel{\mathscr{\nu}}{\check{\nu}}$ & $\stackrel{\mathscr{\nu}}{\check{\nu}}$ & 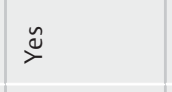 & $\stackrel{2}{2}$ & 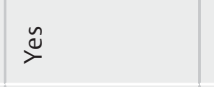 & 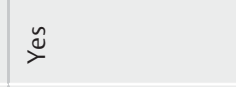 & $\stackrel{\circ}{z}$ \\
\hline 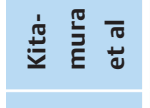 & $\stackrel{y}{\nearrow}$ & 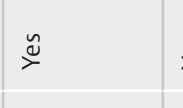 & $\stackrel{0}{0}$ & $\stackrel{0}{=}$ & $\stackrel{2}{z}$ & 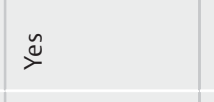 & $\stackrel{0}{\ddot{2}}$ & $\stackrel{2}{z}$ \\
\hline 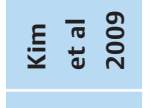 & $\stackrel{\square}{\nearrow}$ & $\stackrel{\check{\nu}}{\check{\nu}}$ & $\mathscr{0}$ & $\cong$ & 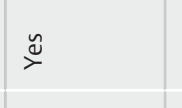 & 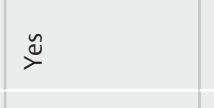 & 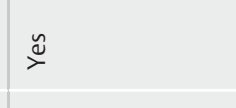 & $\stackrel{\circ}{z}$ \\
\hline 苙要 & 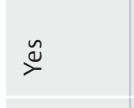 & $\stackrel{0}{2}$ & $\stackrel{0}{x}$ & $\stackrel{\infty}{\infty}$ & $\stackrel{0}{x}$ & 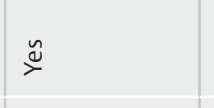 & $\stackrel{y}{y}$ & $\stackrel{8}{z}$ \\
\hline 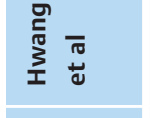 & 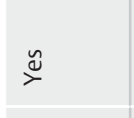 & $\stackrel{0}{0}$ & 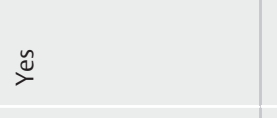 & $\stackrel{\infty}{\infty}$ & 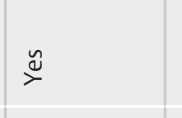 & 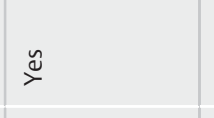 & 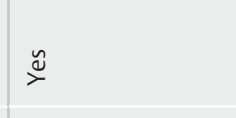 & $\stackrel{2}{z}$ \\
\hline 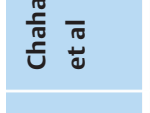 & 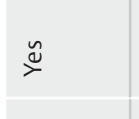 & ֻั & ֶֻ. & $\stackrel{\infty}{=}$ & $\stackrel{8}{z}$ & 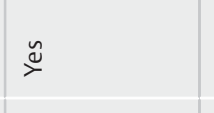 & $\stackrel{\infty}{=}$ & $\stackrel{2}{z}$ \\
\hline 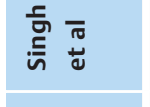 & 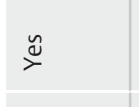 & $\stackrel{y}{\check{\nu}}$ & ֻِّ & 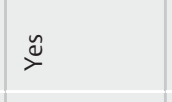 & $\stackrel{8}{z}$ & 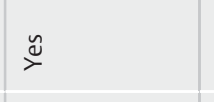 & $\stackrel{\infty}{3}$ & $\stackrel{2}{z}$ \\
\hline 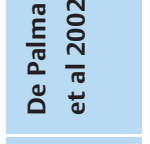 & 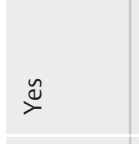 & 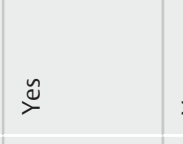 & 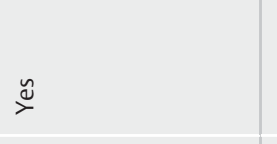 & $\stackrel{y}{0}$ & 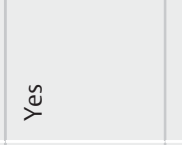 & 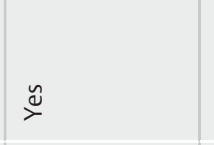 & 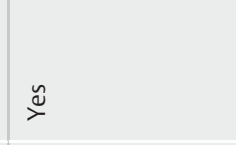 & $\stackrel{̊}{z}$ \\
\hline 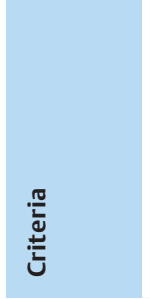 & 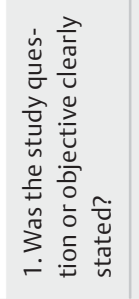 & 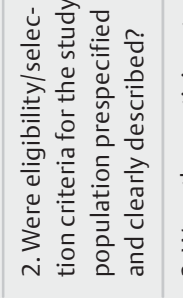 & 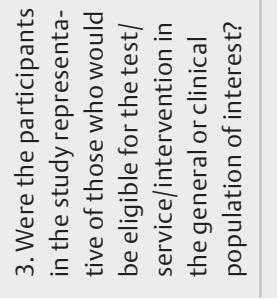 & 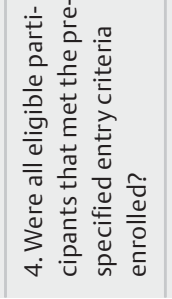 & 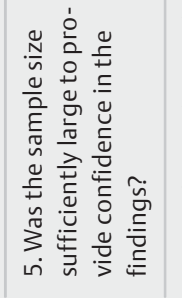 & 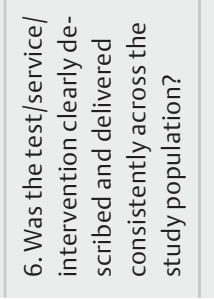 & 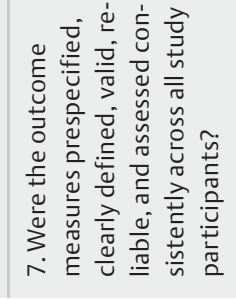 & 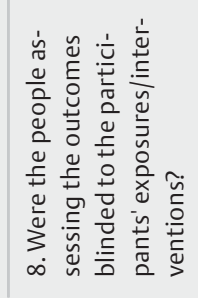 \\
\hline
\end{tabular}




\begin{tabular}{|c|c|c|c|c|c|}
\hline 苂 & $\stackrel{\tilde{\nu}}{\check{\nu}}$ & $\stackrel{\check{\nu}}{\check{\nu}}$ & $\stackrel{\check{\nu}}{\check{\nu}}$ & $\frac{\overleftarrow{z}}{z}$ & : \\
\hline 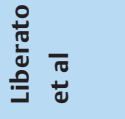 & $\stackrel{\tilde{\Perp}}{\nu}$ & $\stackrel{\check{\nu}}{\nu}$ & $\stackrel{\check{\nu}}{\check{\nu}}$ & $\frac{\leqslant}{z}$ & $\begin{array}{l}\text { "o } \\
\text { o }\end{array}$ \\
\hline $\begin{array}{l}\frac{5}{\overline{0}} \\
\frac{\pi}{\pi} \frac{\pi}{2}\end{array}$ & $\stackrel{\tilde{\nu}}{\check{\nu}}$ & $\stackrel{\tilde{\nu}}{\check{\nu}}$ & $\stackrel{\check{\nu}}{\check{\nu}}$ & $\frac{\pi}{z}$ & $\begin{array}{l}: \\
\end{array}$ \\
\hline 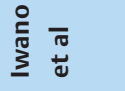 & $\stackrel{\check{\nu}}{\check{\nu}}$ & $\stackrel{\check{\nu}}{\check{\nu}}$ & $\stackrel{\check{\nu}}{\check{\nu}}$ & $\frac{\pi}{z}$ & $\begin{array}{l}\text { : } \\
\text { ò }\end{array}$ \\
\hline 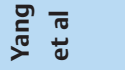 & $\stackrel{\stackrel{a}{\nu}}{2}$ & $\stackrel{0}{z}$ & 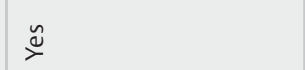 & $\frac{s}{z}$ & 离 \\
\hline 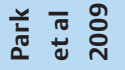 & $\stackrel{\stackrel{\Delta}{\nu}}{2}$ & z & $\stackrel{\tilde{\nu}}{\nu}$ & $\frac{s}{z}$ & 㐫 \\
\hline 弟 & $\stackrel{\nu}{\check{2}}$ & $\stackrel{\Perp}{\nu}$ & 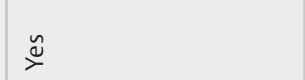 & $\frac{s}{z}$ & $\begin{array}{l}\text { ¿ } \\
\text { o }\end{array}$ \\
\hline$\stackrel{\Xi}{\Xi} \stackrel{m}{\tilde{N}}$ & $\stackrel{\circlearrowright}{\check{\nu}}$ & zo & $\succsim$ & $\frac{s}{z}$ & 施 \\
\hline 汬 & $\stackrel{\circlearrowright}{\nu}$ & $\stackrel{\circlearrowright}{\succ}$ & $\stackrel{\tilde{\nu}}{\nu}$ & $\frac{s}{z}$ & 离 \\
\hline 苞泀 & 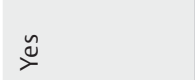 & $\stackrel{\Perp}{\check{\nu}}$ & $\stackrel{\varrho}{\rightleftharpoons}$ & $\frac{s}{z}$ & 㺼 \\
\hline 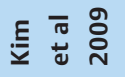 & $\stackrel{\tilde{\nu}}{\succ}$ & $\stackrel{\Perp}{\nu}$ & $\stackrel{\check{\nu}}{\check{\nu}}$ & $\frac{s}{z}$ & ơ \\
\hline 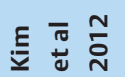 & $\stackrel{\tilde{\nu}}{\nu}$ & $\stackrel{\tilde{\nu}}{\nu}$ & $\stackrel{\nu}{\nu}$ & $\bar{z}$ & نั \\
\hline 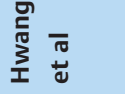 & 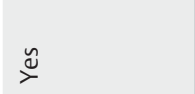 & $\check{\circlearrowright}$ & $\stackrel{\tilde{\nu}}{\check{\nu}}$ & $\frac{l}{z}$ & $\begin{array}{l}\text { : } \\
\text { ¿े }\end{array}$ \\
\hline 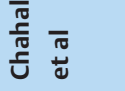 & $\stackrel{\varrho}{\succ}$ & $\stackrel{\Perp}{\succ}$ & $\stackrel{\check{\nu}}{\check{\nu}}$ & $\frac{s}{z}$ & 滑 \\
\hline $\begin{array}{l}\text { 喜 } \\
\text { ज苛 }\end{array}$ & $\stackrel{\nu}{\Perp}$ & $\stackrel{0}{2}$ & 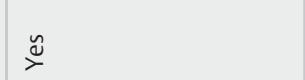 & $\frac{s}{z}$ & $\overline{\tilde{\omega}}$ \\
\hline 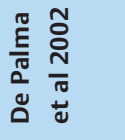 & $\stackrel{\tilde{y}}{\nu}$ & $\stackrel{y}{\nu}$ & $\stackrel{\tilde{\nu}}{\nu}$ & $\frac{s}{z}$ & o. \\
\hline 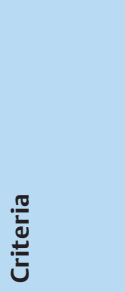 & 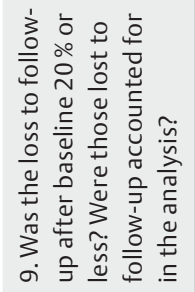 & 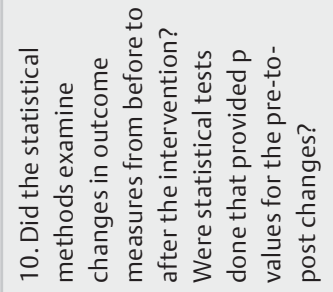 & 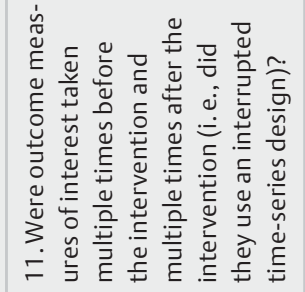 & 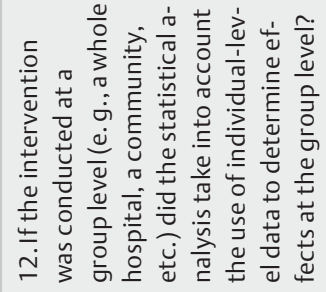 & $\frac{n}{\overrightarrow{5}}$ \\
\hline
\end{tabular}




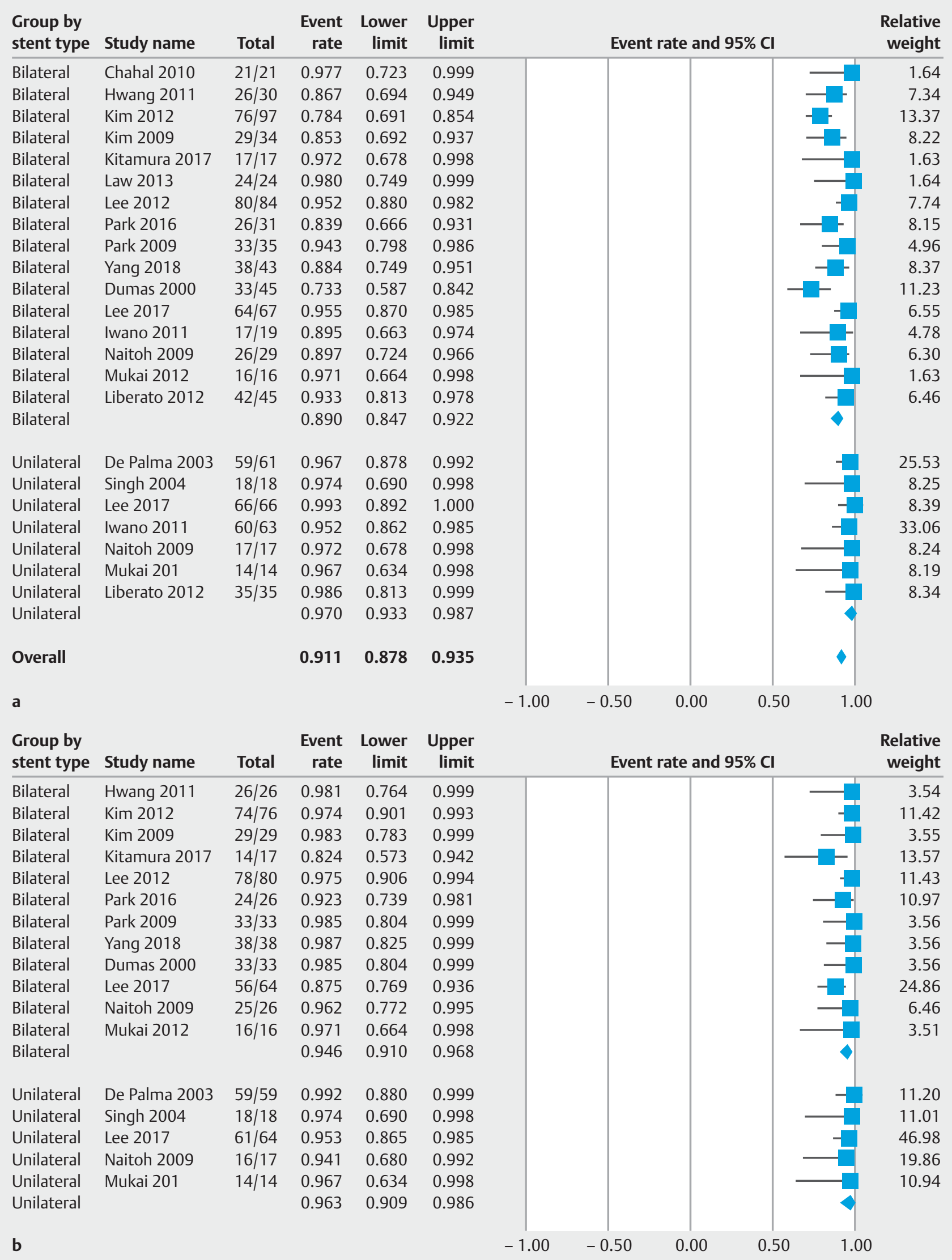

- Fig. 2 Forest plots displaying weighted pool rate of $\mathbf{a}$ technical success and $\mathbf{b}$ functional success. 


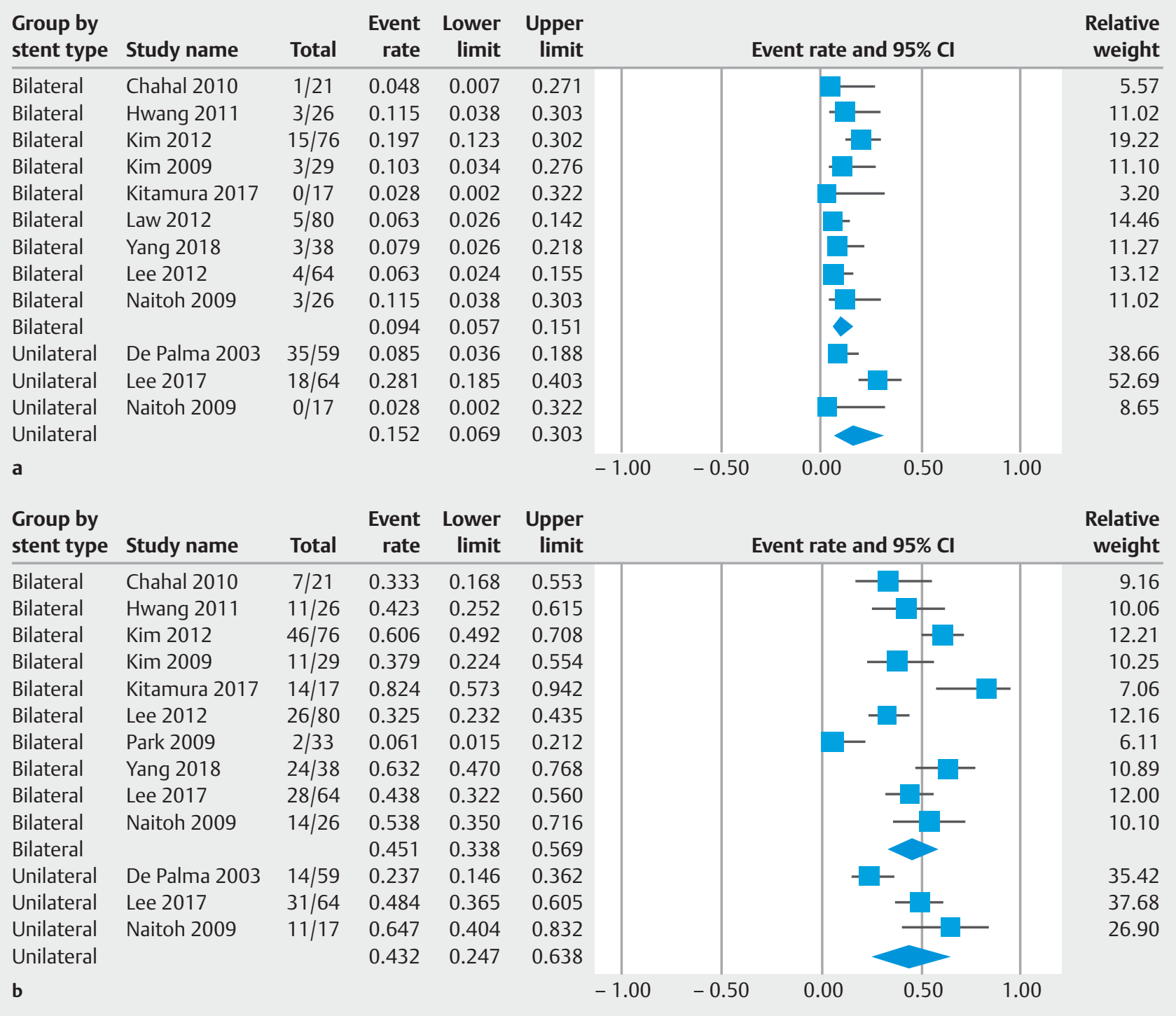

Fig. 3 Forest plots displaying weighted pool rate of a early complications and $\mathbf{b}$ late complication.

of early complication, which occurred in $5.5 \%$ of unilateral and $6.3 \%$ of bilateral groups. In 550 patients, 550 late complications were reported. The WPR of late complication was $43 \%$ (95\%Cl: $25-64 \%)$ in the unilateral group and $45 \%$ (95\% Cl: $33-$ $56 \%$ ) in the bilateral group ( $>$ Fig. 3 b). Obstruction after 30 days occurred in $33.7 \%$ of the unilateral group and $33.1 \%$ in the bilateral group. Fifty-two percent of cases with stent occlusion were managed by insertion of a plastic stent through the metal stent. The remaining patients were managed by SEMS (20\%), percutaneous drainage (24\%), nasobiliary drainage ( $2 \%)$, and cleaning of sludge (2\%). Subgroup analysis demonstrated no statistically significant difference in AEs between unilateral and bilateral metal stent drainage. No mortality related to the procedure was reported.

\section{Discussion}

The majority of patients with malignant hilar biliary obstruction present with non-curative disease which makes palliative care the mainstay of management. Surgical biliary bypass procedures have a higher risk of morbidity and mortality with comparable efficacy to other therapeutic options [40-42]. Therefore, endoscopic and percutaneous stent insertions are frequently used for management of advanced hilar carcinoma. Percutaneous stenting requires external tube placement and is associated with longer hospital stays, which could be less comfortable for patients [7]. Thus, endoscopic biliary drainage has been introduced as a less invasive alternative for palliation of biliary obstruction and is considered as the first choice for management of malignant hilar obstruction [43].

The findings from our meta-analysis revealed that both unilateral and bilateral hilar stenting are associated with comparable efficacy and safety for palliative management of patients 
with malignant hilar obstruction. It should also be noted that a high level of heterogeneity was revealed in the technical success rate of metallic stenting. This could be explained by the different etiologies of malignant hilar obstruction in the patient populations of the included studies. In addition, hilar cholangiocarcinoma, which was the most common etiology among included patients, is anatomically categorized by Bismuth classification into five different groups and the pooled population consisted of patients with various Bismuth classification. Unfortunately, we were unable to perform subgroup analysis based on etiology or Bismuth classification because the included studies did not report their outcome based on these covariates.

Our findings also suggest that the technical success rate of unilateral metallic stenting was significantly higher than that for bilateral metallic stenting. This may be due to the challenges that are associated with insertion of a bilateral metallic stent. Prior studies have also reported on the technical challenges of insertion of a bilateral metal stent, and recommended stricture dilation before stent insertion as a solution, to improve the technical success rate of second metallic stent insertion [44]. In addition, multiple techniques have been described for bilateral stent insertion. Most of them were developed as to "stent-by-stent" or "stent-in-stent" techniques [27]. However, detailed categorization based on different insertion techniques was not possible because the included studies did not report their outcomes based on these categories. Further investigations are warranted to compare outcomes of different insertion techniques and determine the most effective method. In our meta-analysis no significant difference was observed in terms of functional success or early and late complications between the unilateral and bilateral metallic stenting groups. These findings are in contrast with a previous systematic review which showed lower overall, early and late complication rates for bilateral metallic stent [44]. This discrepancy may be explained by the distinct differences in the methodology and execution of these two studies. The mentioned systematic review included published abstracts and no quality assessment was performed for observational studies whereas in our meta-analysis, we excluded published abstracts due to the discrepancy between full-text articles and published abstracts [46, 46]. Small case series were also excluded to decrease the effects of small studies on the results. We also performed a quality assessment for both RCTs and observational studies.

We acknowledge that our study has several limitations. First, the majority of included studies were observational, which could raise concerns about presence of selection bias. Second, we could not categorize our results based on type of malignant neoplasm or bismuth classification because the included studies did not report patient outcomes based on their etiologies and classification. Finally, included studies used different types, brands, and sizes of stents, which couln increase the level of heterogeneity of our results.

\section{Conclusion}

In conclusion, the findings from our study suggest that both unilateral and bilateral drainage of malignant hilar obstruction can effectively relieve biliary obstruction. Although unilateral metallic stent insertion had a significantly higher technical success rate, no significant difference was observed in terms of functional success rates. Early and late complication rates were also comparable between the two groups.

\section{Competing interests}

Dr. Kalloo is a founding member of, and equity holder in, Apollo Endosurgery. Dr. Kumbhari is a consultant for Boston Scientific, Reshape Life Science, Medtronic, Pentax Medical and Apollo Endosurgery. Dr. Singh is a consultant for Akeca Therapeutics, AbbVie and Ariel Precision Medicine. Dr. Khashab is a consultant for Boston Scientific, Olympus and Medtronic and is on the medical advisory board for Boston Scientific and Olympus.

\section{References}

[1] Bismuth H, Castaing D, Traynor O. Resection or palliation: priority of surgery in the treatment of hilar cancer. World J Surg 1988; 12: 39-47

[2] Hong W, Sun X, Zhu Q. Endoscopic stenting for malignant hilar biliary obstruction: should it be metal or plastic and unilateral or bilateral? Eur J Gastroenterol Hepatol 2013; 25: 1105-1112

[3] Freeman ML, Sielaff TD. A modern approach to malignant hilar biliary obstruction. Rev Gastroenterol Disord 2003; 3: 187-201

[4] Perdue DG, Freeman ML, DiSario JA et al. Plastic versus self-expanding metallic stents for malignant hilar biliary obstruction: a prospective multicenter observational cohort study. J Clin Gastroenterol 2008; 42: 1040-1046

[5] Fukasawa M, Takano S, Shindo $\mathrm{H}$ et al. Endoscopic biliary stenting for unresectable malignant hilar obstruction. Clin J Gastroenterol 2017; 10: $485-490$

[6] Kato H, Tsutsumi K, Kawamoto H et al. Current status of endoscopic biliary drainage for unresectable malignant hilar biliary strictures. World J Gastrointest Endosc 2015; 7: 1032

[7] Paik WH, Park YS, Hwang J-H et al. Palliative treatment with self-expandable metallic stents in patients with advanced type III or IV hilar cholangiocarcinoma: a percutaneous versus endoscopic approach. Gastrointest Endosc 2009; 69: 55-62

[8] $\mathrm{Kim} \mathrm{JH}$. Endoscopic stent placement in the palliation of malignant biliary obstruction. Clin Endosc 2011; 44: 76

[9] Cheng JL, Bruno MJ, Bergman J] et al. Endoscopic palliation of patients with biliary obstruction caused by nonresectable hilar cholangiocarcinoma: efficacy of self-expandable metallic Wallstents. Gastrointest Endosc 2002; 56: 33-39

[10] Lee TH, Moon JH, Park S-H. Bilateral metallic stenting in malignant hilar obstruction. Clin Endosc 2014; 47: 440

[11] Kogure H, Isayama H, Kawakubo K et al. Endoscopic bilateral metallic stenting for malignant hilar obstruction using newly designed stents. J Hepatobiliary Pancreat Sci 2011; 18: 653-657

[12] Liberati A, Altman DG, Tetzlaff ] et al. The PRISMA statement for reporting systematic reviews and meta-analyses of studies that evalu- 
ate health care interventions: explanation and elaboration. PLoS Med 2009; 6: e1000100

[13] Stroup DF, Berlin JA, Morton SC et al. Meta-analysis of observational studies in epidemiology: a proposal for reporting. JAMA 2000; 283: 2008-2012

[14] NIH. Quality Assessment Tool for Before-After Studies with No Control Group. National Institutes of Health Web site.http://www.nhlbi. nih.gov/health-pro/guidelines/in-develop/cardiovascular-risk-reduction/tools/before-after

[15] Higgins JP, Thompson SG, Deeks J] et al. Measuring inconsistency in meta-analyses. BMJ 2003; 327: 557

[16] Armitage P, Berry G, Matthews JNS. Statistical methods in medical research. John Wiley \& Sotns; 2008

[17] DerSimonian R, Laird N. Meta-analysis in clinical trials. Control Clin Trials 1986; 7: 177-188

[18] Riley RD, Higgins JP, Deeks JJ. Interpretation of random effects metaanalyses. BMJ 2011; 342: d549

[19] Begg CB, Mazumdar M. Operating characteristics of a rank correlation test for publication bias. Biometrics 1994; 50: 1088-1101

[20] Hsieh J, Thosani A, Grunwald M et al. Serial insertion of bilateral uncovered metal stents for malignant hilar obstruction using an $8 \mathrm{Fr}$ biliary system: a case series of 17 consecutive patients. Hepatobiliary Surg Nutr 2015; 4: 348

[21] Lee TH, Park DH, Lee SS et al. Technical feasibility and revision efficacy of the sequential deployment of endoscopic bilateral side-by-side metal stents for malignant hilar biliary strictures: a multicenter prospective study. Dig Dis Sci 2013; 58: 547-555

[22] Lee T, Moon J, Kim J et al. Primary and revision efficacy of cross-wired metallic stents for endoscopic bilateral stent-in-stent placement in malignant hilar biliary strictures. Endoscopy 2013; 45: 106-113

[23] Chahal P, Baron TH. Expandable metal stents for endoscopic bilateral stent-within-stent placement for malignant hilar biliary obstruction. Gastrointest Endosc 2010; 71: 195-199

[24] Iwano H, Ryozawa S, Ishigaki N et al. Unilateral versus bilateral drainage using self-expandable metallic stent for unresectable hilar biliary obstruction. Dig Endosc 2011; 23: 43-48

[25] Kim DU, Kang DH, Kim GH et al. Bilateral biliary drainage for malignant hilar obstruction using the 'stent-in-stent'method with a Ystent: efficacy and complications. Eur J Gastroenterol Hepatol 2013; 25: 99-106

[26] Kitamura K, Yamamiya A, Ishii Y et al. Side-by-side partially covered self-expandable metal stent placement for malignant hilar biliary obstruction. Endosc Int Open 2017; 5: E1211

[27] Law R, Baron TH. Bilateral metal stents for hilar biliary obstruction using a $6 \mathrm{Fr}$ delivery system: outcomes following bilateral and side-byside stent deployment. Dig Dis Sci 2013; 58: 2667-2672

[28] Liberato MJA, Canena JMT. Endoscopic stenting for hilar cholangiocarcinoma: efficacy of unilateral and bilateral placement of plastic and metal stents in a retrospective review of 480 patients. BMC Gastroenterol 2012; 12: 103

[29] Naitoh I, Ohara H, Nakazawa T et al. Unilateral versus bilateral endoscopic metal stenting for malignant hilar biliary obstruction. J Gastroenterol Hepatol 2009; 24: 552-557

[30] Park JM, Lee SH, Chung KH et al. Endoscopic bilateral stent-in-stent placement for malignant hilar obstruction using a large cell type stent. Hepatobiliary Pancreat Dis Int 2016; 15: 633-639
[31] Dumas R, Demuth N, Buckley M et al. Endoscopic bilateral metal stent placement for malignant hilar stenoses: identification of optimal technique. Gastrointest Endosc 2000; 51: 334-338

[32] Lee TH, Kim TH, Moon JH et al. Bilateral versus unilateral placement of metal stents for inoperable high-grade malignant hilar biliary strictures: a multicenter, prospective, randomized study (with video). Gastrointest Endosc 2017; 86: 817-827

[33] Mukai T, Yasuda I, Nakashima M et al. Metallic stents are more efficacious than plastic stents in unresectable malignant hilar biliary strictures: a randomized controlled trial. J Hepatobiliary Pancreat Sci 2013; 20: 214-222

[34] Hwang JC, Kim JH, Lim SG et al. Y-shaped endoscopic bilateral metal stent placement for malignant hilar biliary obstruction: prospective long-term study. Scand J Gastroenterol 2011; 46: 326-332

[35] Kim JY, Kang DH, Kim HW et al. Usefulness of slimmer and open-celldesign stents for endoscopic bilateral stenting and endoscopic revision in patients with hilar cholangiocarcinoma (with video). Gastrointest Endosc 2009; 70: 1109-1115

[36] Park DH, Lee SS, Moon JH et al. Newly designed stent for endoscopic bilateral stent-in-stent placement of metallic stents in patients with malignant hilar biliary strictures: multicenter prospective feasibility study (with videos). Gastrointest Endosc 2009; 69: 1357-1360

[37] Yang M], Kim JH, Hwang JC et al. Prospective multicenter study on the challenges inherent to using large cell-type stents for bilateral stentin-stent placement in patients with inoperable malignant hilar biliary obstruction. Gut Liver 2018; 12: 722-727

[38] De Palma GD, Pezzullo A, Rega M et al. Unilateral placement of metallic stents for malignant hilar obstruction: a prospective study. Gastrointest Endosc 2003; 58: 50-53

[39] Singh V, Singh G, Verma G et al. Contrast-free unilateral endoscopic palliation in malignant hilar biliary obstruction: New method. J Gastroenterol Hepatol 2004; 19: 589-592

[40] De Palma GD, Galloro G, Siciliano S et al. Unilateral versus bilateral endoscopic hepatic duct drainage in patients with malignant hilar biliary obstruction: results of a prospective, randomized, and controlled study. Gastrointest Endosc 2001; 53: 547-553

[41] Guthrie C, Banting S, Garden O et al. Segment III cholangiojejunostomy for palliation of malignant hilar obstruction. Br J Surg 1994; 81: 1639-1641

[42] Vauthey JN, Blumgart LH. Recent advances in the management of cholangiocarcinomas. In: Seminars in liver disease. Thieme Medical Publishers, Inc; 1994: 109-114

[43] Speer A, Christopher R, Russell G et al. Randomised trial of endoscopic versus percutaneous stent insertion in malignant obstructive jaundice. Lancet 1987; 330: 57-62

[44] Puli SR, Kalva N, Pamulaparthy SR et al. Bilateral and unilateral stenting for malignant hilar obstruction: a systematic review and meta-analysis. Indian J Gastroenterol 2013; 32: 355-362

[45] Scherer RW, Langenberg P, Von Elm E. Full publication of results initially presented in abstracts. Cochrane Database Syst Rev 2018; 11: MR000005

[46] Taddio A, Pain T, Fassos FF et al. Quality of nonstructured and structured abstracts of original research articles in the British Medical Journal, the Canadian Medical Association Journal and the Journal of the American Medical Association. CMAJ 1994; 150: 1611 